Pacific Journal of Mathematics

COVARIANT REPRESENTATIONS OF INFINITE TENSOR 


\title{
COVARIANT REPRESENTATIONS OF INFINITE TENSOR PRODUCT ALGEBRAS
}

\author{
Richard H. Herman and Michael C. Reed
}

\begin{abstract}
In this paper myriad covariant representations of a class of $C^{*}$-algebras and automorphism groups are constructed. The Hilbert spaces on which the representations are realized have an unusual structure: they are direct integrals of measurable families $\mathscr{S}(\cdot)$ of Hilbert spaces over the spectrum of an abelian subalgebra of the $C^{*}$-algebra; the fibre spaces $\mathscr{S}(\zeta)$ are (in general) different separable subspaces of inseparable infinite tensor product spaces. The representors of the algebra and the unitary representors of the group do not decompose but act both in the fibres and on the underlying spectrum. Cases covered by this construction include the quasifree automorphisms of the Clifford algebra which leave a given basis fixed and the automorphism corresponding to charge conjugation.
\end{abstract}

The problem of constructing representations of a $C^{*}$-algebra $\mathfrak{A}$ in which certain automorphism groups are unitarily implementable, has received considerable attention. Not only are the questions involved quite natural from a mathematical point of view, but there are important applications in quantum field theory and statistical mechanics. The usual approach to the problem is to use a fixed point theorem to prove the existence of a state, $\omega$ in $\mathfrak{A}^{*}$, which is invariant under the contragradient action of the automorphism group on $\mathfrak{A}^{*}$. The GNS construction (13) then guarantees a covariant representation of $\mathfrak{A}$, i.e., one in which the automorphisms are unitarily implementable. The difficulty with this procedure in applications is that one has very little analytical control over the resulting representation. There are two reasons for this: First, the properties of the representation will depend strongly on which fixed point is chosen and the fixed point theorem may not give any convenient way of distinguishing them. Secondly, the nature of the GNS construction itself is such that it is difficult to carry analytical information from the algebra (or the original representation) to the new representation. What is needed are special methods (necessarily suited to special kinds of algebras and automorphism groups) of constructing covariant representations so that the objects in the representation are given more or less explicitly. In this paper we give such a special method. The idea is to begin with a very simple kind of invariant state (a product state). We first realize the corresponding representation of $\mathfrak{A}$ in such a way that the properties of the representation which make it covariant are explicitly 
displayed. We then perturb the representation to obtain other nonequivalent (and nonproduct) representations which are also covariant.

Let $\mathfrak{2}$ be a separable $C^{*}$-algebra which can be written as an infinite tensor product of $C^{*}$-algebras, $\mathfrak{N}_{i}$, viz. $\mathfrak{N}=\check{\otimes} \mathfrak{N}_{i}$ (see [4]). For the purpose of exposition we restrict ourselves to the case where each $\mathfrak{U}_{i}$ is a subalgebra of a matrix algebra. Other cases are covered briefly in $\S \mathrm{VI}$ and for these reasons our proofs are couched in general terminology. Suppose that $\mathfrak{B}_{i}$ is an abelian subalgebra of $\mathfrak{A}_{i}$. Let $N\left(\mathfrak{B}_{i}\right)$ be the group of unitary operators, $U \in \mathfrak{N}_{i}$, such that $U \mathfrak{B}_{i} U^{-1}=\mathfrak{B}_{i}$. We call $N\left(\mathfrak{B}_{i}\right)$ the normalizer of $\mathfrak{B}_{i}$.

DEFINITION. The subalgebra $\mathfrak{B}_{i}$ of $\mathfrak{A}_{i}$ is called regular if the linear span of $N\left(\mathfrak{B}_{i}\right)$ is all of $\mathfrak{N}_{i}$. We assume regularity throughout.

As an example we note that every maximal abelian subalgebra of a full matrix algebra is regular.

Returning to our previous notation, let $\mathfrak{B}$ denote $\check{\otimes} \mathfrak{B}_{i}$. We call

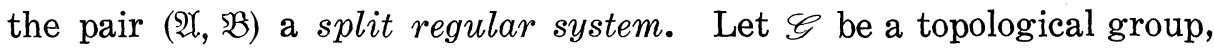
$\tau_{g}, g \in \mathscr{C}$, a representation of $\mathscr{C}$ by automorphisms of $\mathfrak{A}$. We assume that $\tau_{g}$ has the following properties:

(i) $\tau_{g}$ is strongly continuous, i.e., if $g_{n} \stackrel{\leftrightarrow}{\longrightarrow} g$ then $\left\|\tau_{g_{n}}(A)-\tau_{g}(A)\right\|_{\mathscr{\Re}} \rightarrow 0$ for each $A \in \mathfrak{X}$.

(ii) $\tau_{g}=\otimes \tau_{g}^{n}$. That is, there exist automorphisms $\tau_{g}^{n}$ of $\mathfrak{U}_{n}$ so that

$$
\tau_{g}\left(\bigotimes_{n=1}^{N} A_{n} \bigotimes_{n>N} I\right)=\bigotimes_{n=1}^{N} \tau_{g}^{n}\left(A_{k}\right) \bigotimes_{n>N} I
$$

(iii) $\tau_{g}^{n}: \mathfrak{B}_{n} \rightarrow \mathfrak{B}_{n}$.

We will call the triple $\left(\mathfrak{A}, \mathfrak{B}, \tau_{\mathscr{S}}\right)$ a split regular $\mathscr{G}$-system. In Section II we show how to explicitly construct numerous covariant representations of split regular $\mathscr{G}$-systems, i.e., representations of $\mathfrak{A}$ in which the automorphisms, $\tau_{g}$, are unitarily implementable. These representations are not (in general) product representations.

In $\S$ III we discuss some examples of the construction including the quasi-free automorphisms of the Clifford algebra which leave a basis fixed and the charge conjugation automorphism. Both of these groups of automorphisms arise in quantum field theory.

The construction of $\S I I$ uses as its starting point a $\mathfrak{B}$-faithful invariant product state. In $\S I V$ we show that all representations genetated by invariant (possibly) nonfaithful product states occur as subrepresentations of the ones constructed in $\S$ II.

The construction of $\S$ II will not (in general) yield all covariant representations of the system ( $\left.\mathfrak{A}, \mathfrak{B}, \tau_{\mathscr{S}}\right)$. This is because of the special nature of the fibres (infinite tensor product spaces) and the fibre maps. However, in the case where each $\mathfrak{B}_{i}$ is maximal abelian in $\mathfrak{A}_{i}$, one can 
use the theory of cross products to prove that every covariant representation can be decomposed on a direct integral of Hilbert spaces over the spectrum of $\mathfrak{B}$ with some fibres and some fibre maps. This is done in $\S \mathrm{V}$. Section VI is a general discussion which compares the methods we use in $\S I I$ with more "orthodox" ones. In addition, a few brief remarks about the case where $\mathfrak{A}_{i}$ is infinite dimensional, are given.

\section{The construction.}

The $\omega$-bundle. Let $\omega=\otimes \omega_{n}$ be a product state which is invariant under the automorphism group $\left\{\tau_{g}: g \in \mathscr{G}\right\}$ and which is faithful on $\mathfrak{B}$. The GNS construction using $\omega_{n}$ then gives us a representation $\pi_{n}$ of $\mathfrak{2}_{n}$ on a Hilbert space $H_{n}$. (Here $\pi_{n}$ is nothing but $A \rightarrow$ $A \otimes I$ where $I$ is the identity on a finite dimensional Hilbert space.) Moreover, there is a cyclic vector $x_{n}$, of norm one such that $\omega_{n}(A)=$ $\left(\pi_{n}(A) x_{n}, x_{n}\right)$. The invariance of $\omega_{n}$ gives us a unitary representation of $\mathscr{G}$ on $H_{n}$ such that $U_{g}^{n} x_{n}=x_{n}$ and $\pi_{n}\left(\tau_{g}^{n}(A)\right)=U_{g}^{n} \pi_{n}(A) U_{g^{-1}}^{n}$ for all $g \in \mathscr{C}$.

Now, following the usual procedure [1], we decompose the space $H_{n}$ with respect to the abelian algebra $\pi_{n}\left(\mathfrak{B}_{n}\right)$. Thus we pick a vector $y_{n}$, of norm one, which is cyclic for $\pi_{n}\left(\mathfrak{B}_{n}\right)^{\prime}$. If $Z_{n}$ denotes the spectrum of $\mathfrak{B}_{n}$, then the state $\left(\pi_{n}(\cdot) y_{n}, y_{n}\right)$ defines a measure, $\mu_{n}$, on $Z_{n}$. The measure $\mu_{n}$ has, support equal to $Z_{n}$, because $\omega_{n}$ is faithful on $\mathfrak{B}_{n}$. In this case where $Z_{n}$ is finite this means a nonzero mass is assigned to each point. The measure $\mu_{n}$ gives the decomposition, $H_{n}=\int_{z_{n}} \mathscr{H}_{n}\left(\zeta^{n}\right) d \mu_{n}\left(\zeta^{n}\right)$ and since $\omega_{n}$ is faithful on $\mathfrak{B}_{n}, x_{n}\left(\zeta^{n}\right) \neq 0$ for all $\zeta^{n} \in Z_{n}$. This last remark allows us to replace $\mu_{n}$ with an equivalent measure and assume that $\left\|x_{n}\left(\zeta^{n}\right)\right\|_{H_{n}\left(\zeta^{n}\right)}=1$ for all $\zeta^{n} \in Z_{n}$. Having done this for each $n$, we define the $\omega$-bundle to be the pair $\left(\times Z_{n}, \mathscr{S}_{\mathcal{C}}^{\omega}(\cdot)\right)$, where $\times Z^{n}$ is the cartesian product of the $Z_{n}$ and for $\zeta=\left(\zeta^{1}, \zeta^{2}, \cdots\right) \in Z_{n}, \mathfrak{S E}^{\omega}(\zeta)=$ $H\left(\otimes_{n=1}^{\infty} x_{n}\left(\zeta^{n}\right)\right)$, the separable subspace of $\otimes \mathscr{H}_{n}\left(\zeta^{n}\right)$ generated by the $c_{0}$-vector $\otimes_{n=1}^{\infty} x_{n}\left(\zeta^{n}\right)$. We denote by $\Omega_{0}(\cdot)$ the section of $\left(\times Z_{n}, \mathfrak{S}^{\omega}(\cdot)\right)$ whose value at $\zeta$ is $\Omega_{0}(\zeta)=\bigotimes_{n=1}^{\infty} x_{n}\left(\zeta^{n}\right)$.

The action of the algebra. We first examine one component of the tensor product. Since the normalizer of $\mathfrak{B}_{n}$ spans $\mathfrak{U}_{n}$, the set $\left\{\pi_{n}(V) x_{n}\right\}$ as $V$ ranges over $N\left(\mathfrak{B}_{n}\right)$ is total in $H_{n}$. Hence $\left\{\pi_{n}(V) x_{n}\left(\zeta^{n}\right)\right\}$ is total in $\mathscr{H}_{n}\left(\zeta^{n}\right)$ for all (in general almost all) $\zeta^{n}$ in $Z_{n}$. We may clearly extract a countable set from $\left\{\pi_{n}(V) x_{n}\right\}$ with the same property.

The algebra $\mathfrak{B}_{n}$, and hence $\pi_{n}\left(\mathfrak{B}_{n}\right)$ may be regarded as $C\left(Z_{n}\right)$. If $V_{n} \in N\left(\mathfrak{B}_{n}\right)$, then by the Banach-Stone theorem, there is a one-to-one 


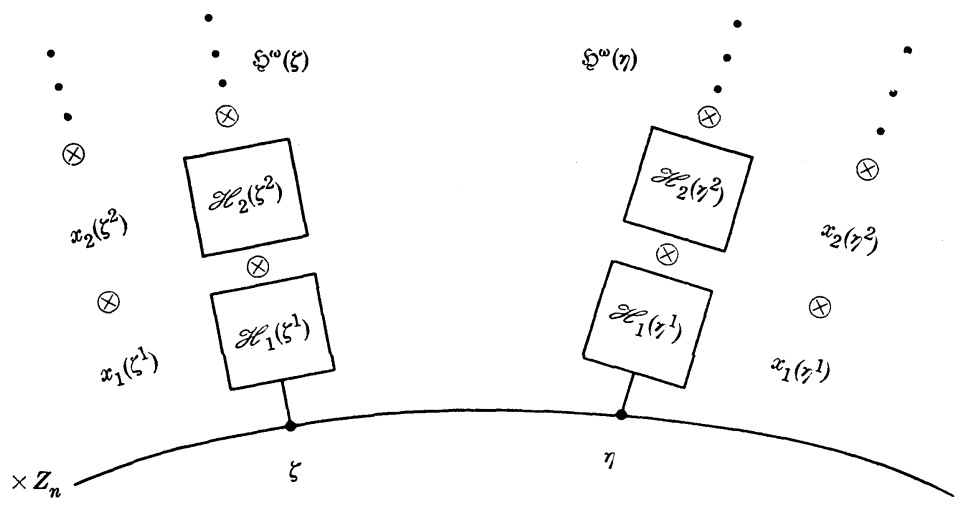

FiguRE 1. The $\omega$-bundle

map, $v_{n}$, of $Z_{n}$ onto $Z_{n}$ such that

$$
\left(\pi_{n}\left(V_{n}\right) f\left(\pi\left(V_{n}^{-1}\right)\right)\left(\zeta^{n}\right)=f\left(v_{n}^{-1} \zeta^{n}\right), \zeta^{n} \in Z_{n}, f \in C\left(Z_{n}\right) .\right.
$$

We refer to $v_{n}^{-1}$ as the action induced on $Z_{n}$ by $V_{n}$, and denote by $\mathscr{U}_{n}$ the group of all such $v_{n}^{-1}$. The weak direct product of the groups $\mathscr{U}_{n}$ forms a group, $\mathscr{U}$, of transformations of $Z=\times Z_{n}$.

Since $\mu_{n}$ is quasi-invariant under the action of each $v_{n}^{-1}$ in $\mathscr{U}_{1}$, a theorem of Guichardet [5] allows us to conclude the existence of unitary fibre maps

$$
\Theta_{v_{n}^{-1}, \zeta^{n}}^{n}: \mathscr{H}_{n}\left(v_{n}^{-1} \zeta^{n}\right) \longrightarrow \mathscr{H}_{n}\left(\zeta^{n}\right)
$$

such that $\pi_{n}\left(V_{n}\right)$ is given explicitly by

$$
\left(\pi_{n}\left(V_{n}\right) x\right)\left(\zeta^{n}\right)=r_{v_{n}}\left(v_{n}^{-1} \zeta^{n}\right)^{-1 / 2} \Theta_{v_{n}^{-1}, \zeta^{n}}\left(x\left(v_{n}^{-1} \zeta^{n}\right)\right)
$$

where $r_{v_{n}}\left(\zeta^{n}\right)$ is the Radon-Nikodym derivative $\left[d \mu_{n}\left(v_{n} \cdot\right) / d \mu_{n}(\cdot)\right]\left(\zeta^{n}\right)$.

For each sequence $V_{n}, n=1,2, \cdots, N, V_{n} \in N\left(\mathfrak{B}_{n}\right)$, we have a natural action $v^{-1}$ on $Z$ given by $v^{-1 \zeta}=\left(v_{1}^{-1} \zeta^{1}, \cdots, v_{n}^{-1} \zeta^{n}, \zeta^{n+1}, \cdots\right)$ and a well-defined family

$$
\Theta_{v^{-1, \zeta}}: \mathfrak{S E}^{\omega}\left(v^{-1} \zeta\right) \longrightarrow \mathfrak{S}^{\omega}(\zeta)
$$

of fibre maps of $\mathfrak{S E}^{\omega}(\cdot)$ where

$$
\Theta_{v^{-1, \zeta}}=\left(\bigotimes_{n=1}^{N} \Theta_{v_{n}^{-1}, \zeta^{n}}\right) \otimes\left(\bigotimes_{n>N} I\right) \text {. }
$$

As remarked above, the set

$$
\left\{\left(\pi_{n}\left(V_{n}\right) x_{n}\right)\left(\zeta^{n}\right) ; V_{n} \in N\left(\mathfrak{B}_{n}\right)\right\}
$$

is total in $\mathscr{H}_{n}\left(\zeta^{n}\right)$. Since $r_{v_{n}}(\cdot)$ is nowhere zero on $Z_{n}$, the set 
$\left\{\Theta_{v_{n}^{-1}, \zeta^{n}}^{n}\left(x_{n}\left(v_{n}^{-1} \zeta^{n}\right)\right) ; v_{n}^{-1} \in \mathscr{C}_{n}\right\}$ is also total in $\mathscr{H}_{n}\left(\zeta^{n}\right)$. Therefore

$$
\left\{\Theta_{v^{-1, \zeta}}\left(\left(v^{-1 \zeta}\right)\right) ; v \in \mathscr{\mathscr { C }}\right\}
$$

is total in $\mathfrak{S C}^{\omega}(\zeta)$. Thus the finite linear combinations of sections of the form

$$
\Omega_{v}(\cdot)=\Theta_{v^{-1}, .} \Omega_{0}\left(v^{-1} \cdot\right)
$$

form a measurable field of cross-sections, $\mathbb{S}$, for $\left(Z, \mathfrak{S C}^{\omega}(\cdot)\right)$. The measurability follows from the fact that for any two such cross-sections, $\Omega_{v}(\cdot)$ and $\Omega_{v^{\prime}}(\cdot),\left(\Omega_{v}(\zeta), \Omega_{v^{\prime}}(\zeta)\right)_{\mathfrak{\xi}^{\omega}(\zeta)}$ is a finite sum of finite products of measurable functions.

The action of the group. We return again to the component algebra, $\mathfrak{A}_{n}$. Since $\omega_{n}$ is invariant, there is a unitary representation, $U_{g}^{n}$ of $\mathscr{G}$ on $\mathscr{H}_{n}$ so that

$$
\pi_{n}\left(\tau_{g}(A)\right)=U_{g}^{n} \pi_{n}(A) U_{g^{-1}}^{n} .
$$

We again consider the decomposition

$$
H_{n}=\int_{z_{n}} \mathscr{H}_{n}\left(\zeta^{n}\right) d \mu_{n}\left(\zeta^{n}\right)
$$

discussed above. Since $\tau_{g}: \mathfrak{B}_{n} \rightarrow \mathfrak{B}_{n}$ we can conclude from the Banach Stone theorem that for each $g \in \mathscr{G}$ there is a one-to-one transformation (which we denote by $g_{n}$ ) on $Z_{n}$ so that

$$
\left(\tau_{g} f\right)\left(\zeta^{n}\right)=U_{g}^{n} f\left(\zeta^{n}\right) U_{g^{-1}}^{n}=f\left(g_{n}^{-1} \zeta^{n}\right) .
$$

Furthermore, the Guichardet theorem used above again shows the existence of fibre maps

$$
\Xi_{g_{n}^{-1}, \zeta}^{n}: \mathscr{H}_{n}\left(g_{n}^{-1} \zeta^{n}\right) \longrightarrow \mathscr{H}_{n}\left(\zeta^{n}\right)
$$

such that

$$
\left(U_{g}^{n} x\right)(\zeta)=r_{g_{n}}\left(g_{n}^{-1} \zeta^{n}\right)^{-1 / 2} \Xi_{g_{n}^{-1}, \zeta^{n}}^{n}\left(x\left(g_{n}^{-1} \zeta^{n}\right)\right)
$$

for each $x \in H_{n}=\int_{z_{n}} \mathscr{H}_{n}\left(\zeta^{n}\right) d \mu_{n}\left(\zeta^{n}\right)$.

Notice that $\mathscr{G}^{Z_{n}}$ acts in a natural way on $Z=\times Z_{n}$ by $g^{-1}(\zeta)=$ $\left(g_{1}^{-1} \zeta^{1}, g_{2}^{-1} \zeta^{2}, \cdots\right)$. We denote this group of transformations on $Z$ by $\widetilde{G}$. $\widetilde{G}$ is a quotient of $\mathscr{G}$ and may be very small. For example, $g_{n}$ could act like the identity on $Z_{n}$ for each $g \in \mathscr{G}$ and $n$. (This is the case in Example I of $\S$ III.) The action may also be difficult to describe since for a given $g \in \mathscr{G}$, the transformation $g_{n}$ on $Z_{n}$ may act very differently for different $n$. Because $Z_{n}$ is finite the map $g \rightarrow g_{n}$ is a 
homomorphism of $\mathscr{G}$ onto a discrete finite quotient group.

THEOREM 1. Let (î, $\left.\mathfrak{B}, \tau_{\mathscr{S}}\right)$ be a split regular $\mathscr{G}$-system and $\omega=$ $\otimes \omega_{n}$ a $\mathfrak{B}$-faithful product state. Let $\left(Z, \mathfrak{S}^{\omega}(\cdot)\right)$ be the $\omega$-bundle constructed above. Then for every Borel measure, $\nu$, of mass one which is quasi-invariant under $\mathscr{Q}$ and $\tilde{\mathscr{G}}$,

(i) The map $V \rightarrow \pi_{\nu}^{\omega}(V)$ given by

$$
\left[\pi_{\nu}^{\omega}(V) x\right](\zeta)=r_{v}\left(v^{-1 \zeta}\right)^{-1 / 2} \Theta_{v^{-1, \zeta}}\left(x\left(v^{-1 \zeta}\right)\right),
$$

where

$$
V=\bigotimes_{n=1}^{N} V_{n}, V_{n} \in N\left(\mathfrak{B}_{n}\right), r_{v}(\zeta)=[d \nu(v \cdot) / d \nu(\cdot)](\zeta),
$$

extends uniquely to a representation of $\mathfrak{A}$ on $L^{2}\left(Z, \nu, \mathfrak{S E}^{\omega}(\cdot)\right)$. The distinguished section $\Omega_{0}^{\omega}(\cdot)$ is cyclic for $\pi_{\nu}^{\omega}(\mathfrak{U})$.

(ii) In the representation, $\pi_{\nu}^{\omega}$, the automorphisms $\tau_{g}$ are unitarily implemented by

$$
\left(U_{\nu}^{\omega}(g) x\right)(\zeta)=r_{g}\left(g^{-1 \zeta}\right)^{-1 / 2}\left(\bigotimes_{n=1}^{\infty} \Xi_{g_{n}^{-1}, \zeta}^{n}\right) x\left(g^{-1 \zeta}\right) .
$$

If $\mathscr{G}$ is locally compact and second countable then $U_{\nu}^{\omega}(g)$ is strongly continuous.

Proof. As indicated above, the $\Omega_{\nu}^{\omega}(\cdot)$ are a family of measurable sections which are total at each point, so $L^{2}\left(Z, \nu, \mathfrak{F}^{\omega}(\cdot)\right)$ is well-defined. Since $\mathfrak{A}=\breve{\otimes} \mathfrak{U}_{n}$ and $(\mathfrak{A}, \mathfrak{B})$ is split regular it suffices to define $\pi_{\nu}^{\omega}$ on elements of the from $V$ given in (i). If $V_{n}, W_{n} \in N\left(\mathfrak{B}_{n}\right)$ then

$$
\Theta_{\left(v_{n} w_{n}\right)^{-1, \zeta}}^{n}=\Theta_{v_{n}^{-1}, \zeta}^{n} \cdot \Theta_{w_{n}^{-1}, v_{n}^{-1} \zeta}^{n}
$$

since $\pi_{n}^{\omega}$ is a representation of $\mathfrak{U}_{n}$. Thus, if $V, W \in \bigotimes_{n=1}^{N} N\left(\mathfrak{B}_{n}\right)$

$$
\Theta_{(v w)-1, \zeta}=\Theta_{v^{-1, \zeta}} \cdot \Theta_{w^{-1, v_{n}^{-1 \zeta}}} \cdot
$$

This statement, combined with the chain rule for Radon-Nikodym derivatives shows that $\pi_{\nu}^{\omega}(\cdot)$ is in fact a representation of $\mathfrak{A}$. The proof that $\Omega_{0}^{\omega}(\cdot)$ is cyclic is the same as the corresponding proof in [8, Theorem 1].

Consider the fibre map

$$
\Xi_{g^{-1, \zeta}}=\bigotimes_{n=1}^{\infty} \Xi_{g_{n}^{-1}, \zeta^{n}}^{n}
$$

$\Xi_{g^{-1, \zeta}}$ is a well-defined map from $\mathfrak{S c}^{\omega}\left(g^{-1} \zeta\right)$ to $\mathfrak{F}^{\omega}(\zeta)$ since it takes the $c_{0}$-vector $\Omega_{0}^{\omega}\left(g^{-1 \zeta}\right)$, which generates $\mathfrak{S}^{\omega}\left(g^{-1} \zeta\right)$, into $\Omega_{0}^{\omega}(\zeta)$, the $c_{0}$-vector which generates $\mathcal{G}^{\omega}(\zeta)$ (this follows from the invariance of $x_{n} \in \mathscr{H}_{n}$ under $\left.U_{g}^{n}\right)$. The fact that $U_{\nu}^{n}(g)$ is a representation of $\mathscr{G}$ follows from the fact that the $\Xi_{g_{n}^{-1}, \zeta n}^{n}$ compose correctly (and therefore so do the fibre 
maps $\left.\Xi_{g^{-1, \zeta}}\right)$ and the chain rule for $r_{v}(\cdot)$.

It remains to prove that the representation of $\mathscr{G}$ given by $U_{\nu}^{\omega}(g)$ is strongly continuous. The section $\Omega_{0}^{w}(\cdot)$ is cyclic and thus it suffices to prove strong continuity on the dense set of elements $\left\{\pi_{\nu}^{\omega}(A) \Omega_{0}^{\omega} ; A \in \mathfrak{N}\right\}$. Now

$$
\begin{aligned}
U_{\nu}^{\omega}(g) \pi_{\nu}^{\omega}(A) \Omega_{0}^{\omega} & =U_{\nu}^{\omega}(g) \pi_{\nu}^{\omega}(A) U_{\nu}^{\omega}\left(g^{-1}\right) U_{\nu}^{\omega}(g) \Omega_{0}^{\omega} \\
& =\pi_{\nu}^{\omega}\left(\tau_{g}(A)\right) U_{\nu}^{\omega}(g) \Omega_{0}^{\omega}
\end{aligned}
$$

Since $\left\|\tau_{g}(A)-A\right\| \rightarrow 0$ as $g \rightarrow e$, it suffices to show the continuity of $U_{\nu}^{\omega}(g)$ on the cyclic vector $\Omega_{0}^{\omega}$. Thus we must show that

$$
\begin{aligned}
\lim _{g \rightarrow e}\left\|U_{\nu}^{\omega}(g) \Omega_{0}^{\omega}-\Omega_{0}^{\omega}\right\| & =\lim _{g \rightarrow e} \int_{Z}\left|r_{g}\left(g^{-1 \zeta}\right)^{-1 / 2}-1\right|^{2} d \nu(\zeta) \\
& =0
\end{aligned}
$$

Let us look at the map from $\mathscr{G} \times Z$ into $Z$ given by $(g, \zeta) \rightarrow g^{-1} \zeta$. This map is bicontinuous. Let $\zeta_{n}=\left(\zeta_{n}^{1}, \zeta_{n}^{2}, \cdots\right)$ converge to $\zeta=$ $\left(\zeta^{1}, \zeta^{2}, \cdots\right)$ and $g_{n} \rightarrow g$. Let a neighbourhood of $g^{-1} \zeta$ be given by the element $B=B_{1} \otimes \cdots \otimes B_{k} \otimes_{n>k} I_{n}$ belonging to $\mathfrak{B}$. The point $\zeta_{n}$ corresponds to the character, $\rho_{n}$, on $B$ given by $\rho_{n}(B)=B_{1}\left(\zeta_{n}^{1}\right) B_{2}\left(\zeta_{n}^{2}\right) \cdots B_{k}\left(\zeta_{n}^{k}\right)$. With this notation we have

$$
\begin{aligned}
\left|g_{n}^{-1} \zeta_{n}(B)-g^{-1} \zeta(B)\right| & =\left|\rho_{n}\left(\tau_{g_{n}}(B)\right)-\rho\left(\tau_{g}(B)\right)\right| \\
& \leqq\left|\rho_{n}\left(\tau_{g_{n}}(B)\right)-\rho_{n}\left(\tau_{g}(B)\right)+\rho_{n}\left(\tau_{g}(B)\right)-\rho\left(\tau_{g}(B)\right)\right| \\
& \leqq|| \tau_{g_{n}}(B)-\tau_{g}(B)||+\left|\left(\rho_{n}-\rho\right)\left(\tau_{g}(B)\right)\right| .
\end{aligned}
$$

Thus choosing $g_{n}$ sufficiently closed to $g$ it follows by the assumed continuity of $\tau_{g}$ that the first term is small. Since $\tau_{g}(B)=\tau_{g}^{1}\left(B_{1}\right) \otimes$ $\cdots \otimes \tau_{g}^{k}\left(B_{k}\right) \otimes_{n>k} I_{n}$ defines a neighbourhood of $\zeta$, the second term may also be made small.

In the terminology of [16], $Z$ is a Borel $\mathscr{G}$-space. Further $Z$ is a standard Borel space. On $L^{2}(Z, \nu)$ define a unitary representation of $\mathscr{G}$ by

$$
\left(U_{g}^{\nu} f\right)(\zeta)=r_{g}\left(g^{-1 \zeta}\right)^{-1 / 2} f\left(g^{-1 \zeta}\right)
$$

Proving that $(*)$ is true is the same as showing $U_{g}^{\nu} f \underset{g \rightarrow e}{\longrightarrow} f$ for $f$ the function identically equal to one. However a stronger result is true. Namely that the above representation on $L^{2}(Z, \nu)$ is strongly continuous. This is the content of Example 2 of $[16$, p. 60]. In fact it

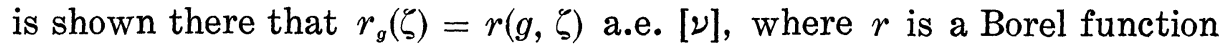
on $\mathscr{G} \times Z$. This makes $\left(U_{g}^{\nu} f, f^{\prime}\right)$ measurable and hence continuous.

A further discussion of the direct integral decomposition used here is given in $\S \mathrm{VI}$. 
III. Examples. In this section we discuss three examples which illustrate the applications of the construction described in \$II.

EXAMPLE 1. (Clifford Algebra, quasi-free automorphisms). Let $\mathfrak{A}$ be the unique $C^{*}$-closure of the Clifford algebra of a real pre-Hilbert space, $H$. $\mathfrak{A}$ can be written, $\mathfrak{A}=\check{\otimes} \mathfrak{N}_{n}$, as the infinite tensor product of two by two matrix algebras, $\mathfrak{A}_{n}$, where each $\mathfrak{A}_{n}$ is generated by 1 and an operator $a_{n}$ satisfying $a_{n}^{2}=0, a_{n}^{*} a_{n}+a_{n} a_{n}^{*}=I$. Let $\mathfrak{B}_{n}$ be the subalgebra of $\mathfrak{U}_{n}$ generated by $I$ and $a_{n}^{*} a_{n}$. $\mathfrak{B}_{n}$ is commutative and if

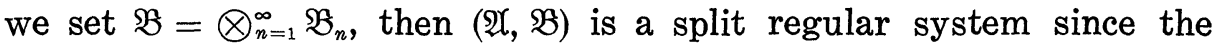
unitary operators $a_{n}+a_{n}^{*}$ and $i\left(a_{n}^{*}-a_{n}\right)$ are in the normalizer of $\mathfrak{B}_{n}$ and, along with $\mathfrak{B}_{n}$, span $\mathfrak{A}_{n}$.

Let $\lambda=\left\{\lambda_{n}\right\}$ be a sequence of real numbers and consider the automorphism $\tau_{\lambda}$ of $\mathfrak{U}$ generated by the transformation

$$
a_{n} \longrightarrow e^{i \lambda_{n}} a_{n} \text {. }
$$

Each such automorphism arises from a unitary operator on a complxification of $H$ which is diagonal with respect to the given basis (the basis that gives rise to the splitting $\left.\mathfrak{A}=\otimes \mathfrak{A}_{n}\right)$. The set of such unitaries on $H$ is a group, $\Lambda$, and for each $\lambda \in \Omega, \tau_{\lambda}$ splits and leaves $\mathfrak{B}_{n}$ pointwise fixed. Thus $\left(\mathfrak{A}, \mathfrak{B}, \tau_{\Lambda}\right)$ is a split regular $\Lambda$-system. For the construction of $\mathfrak{A}$ and a discussion of $\Lambda$ see [8]. Now, let $\omega=$ $\otimes \omega_{n}$ be any $\mathfrak{B}$-faithful product state which is symmetric about the basis (in the terminology of [11]). Then $\omega$ is $\Lambda$-invariant and in this case $\mathfrak{B}$-faithful means that $\omega_{n}\left(a_{n}^{*} a_{n}\right) \neq 0 \neq \omega_{n}\left(a_{n} a_{n}^{*}\right)$.

Thus, we have all the prerequisites for the construction in $\S I I$. Each $Z_{n}$ consists of two points (the spectrum of $\mathfrak{B}_{n}$ ), $Z=\times Z_{n}$, and the fibres, $\mathfrak{S C}^{\omega}(\zeta)$, turn out to be separable subspaces of $\otimes_{n=1}^{\infty} \boldsymbol{C}_{n}^{2}, \boldsymbol{C}_{n}^{2}$ a copy of $C^{2}$; for details see [8]. The point is that once we have constructed the $w$-bunble, $\left(Z, \mathfrak{S}^{\omega}(\cdot)\right)$, then all the representations of $\mathfrak{A}$ on spaces $L^{2}\left(Z, \nu, \mathfrak{F}^{\omega}(\cdot)\right)$, where $\nu$ is quasi-invariant under the induced action of the normalizer on $Z$, allow all the automorphisms, $\tau_{\lambda}$, to be unitarily implemented. There is no $\Lambda$-invariance requirement on $\nu$ since $\mathfrak{B}$ is left pointwise fixed by each $\tau_{\lambda}$. These representations plus the cyclic subrepresentations generated by vectors of the form $\lambda(\cdot) \Omega_{0}^{\omega}(\cdot)$, where $\lambda(\cdot)$ is a $C$-valued $L^{2}$ function on $\{Z, \nu\}$ are all the representations generated by states symmetric about the basis through the GNS construction.

Often one is only interested in a subgroup of $\Lambda$. For example, if $\lambda=\left\{\lambda_{k}\right\}$ is fixed and we consider the automorphisms

$$
a_{n} \rightarrow e^{i \lambda_{n} t} a_{n}, t \in \boldsymbol{R}
$$

then $\left(\mathfrak{A}, \mathfrak{B}, \tau_{\boldsymbol{R}}\right)$ is a split regular $\boldsymbol{R}$-system. If $\lambda_{n}=n$, the corre- 
sponding group is the circle.

ExAmple 2. (Clifford Algebra, Charge Conjugation). Let $\mathfrak{A}$ be the unique $C^{*}$-closure of the Clifford algebra. $\mathfrak{A}$ may be written $\mathfrak{A}=$ $\check{\otimes} \mathfrak{A}_{n}, n=0, \neq 1, \cdots$, where each $\mathfrak{A}_{n}$ is the algebra of all four by four matrices generated by the operators $I, a_{n e}, a_{n e}^{*}, a_{n p}, a_{n p}^{*}$ satisfying

$$
\begin{aligned}
a_{n p}^{2}=0 & =a_{n e}^{2} \\
a_{n p} a_{n e}+a_{n e} a_{n p}=0 & =a_{n e} a_{n p}^{*}+a_{n p}^{*} a_{n e} \\
a_{n e}^{*} a_{n e}+a_{n e} a_{n e}^{*}=I & =a_{n p}^{*} a_{n p}+a_{n p} a_{n p}^{*} .
\end{aligned}
$$

Let $\mathfrak{B}_{n}$ be the abelian algebra generated by $I, a_{n e}^{*} a_{n e}, a_{n p}^{*} a_{n p}$ and $\mathfrak{B}=$ $\check{\otimes} \mathfrak{B}_{n}$. Let $\tau_{c}$ be the unique automorphism of $\mathfrak{A}$ which sends $a_{n e} \rightarrow a_{n p}$ and $a_{n p} \rightarrow a_{n e}$ for each $n$. It is clear that $\tau_{c}$ splits and $\tau_{c} \circ \tau_{c}$ is the identity automorphism so in this case $\mathscr{G}$ is just the cyclic group of order two. The system is regular since $\mathfrak{U}_{n}$ is a full matrix algebra and $\mathfrak{B}_{n}$ is maximal abelian. The operators $a_{n e}^{*}, a_{n p}^{*}$ are related to the creation operators, $\widetilde{a}_{n e}^{*}, \widetilde{a}_{n p}^{*}$, for electrons and positrons of momentum $n$ by formulas

$$
\begin{aligned}
& \tilde{a}_{n e}^{*}=\left(\prod_{k=-(n-1)}^{n-1}\left(I-2 a_{k e}^{*} a_{k e}\right)\right)\left(\prod_{k=-(n-1)}^{n-1}\left(I-2 a_{k p}^{*} a_{k p}\right)\right) a_{n e}^{*} \\
& \tilde{a}_{n p}^{*}=\left(\prod_{k=-(n-1)}^{n-1}\left(I-2 a_{k e}^{*} a_{k e}\right)\right)\left(\prod_{k=-(n-1)}^{n-1}\left(I-2 a_{k p}^{*} a_{k p}\right)\right) a_{n p}^{*}
\end{aligned}
$$

so it is clear that $\widetilde{a}_{n p}^{*}$ and $\widetilde{a}_{n e}^{*}$ (as well as $\widetilde{a}_{n p}$ and $\widetilde{a}_{n e}$ ) are interchanged by $\tau_{c}$.

Briefly, the results of the construction are as follows. For each $n$, the spectrum, $Z_{n}$, of $\mathfrak{B}_{n}$ consists of four points $\zeta_{00}^{n}, \zeta_{10}^{n}, \zeta_{01}^{n}, \zeta_{11}^{n}$ corresponding to the four projections $P_{00}=a_{n e} a_{n e}^{*} a_{n p} a_{n p}^{*}, P_{10}=a_{n e}^{*} a_{n e} a_{n p} a_{n p}^{*}$, $P_{01}=a_{n e} a_{n e}^{*} a_{n p}^{*} a_{n p}, P_{11}=a_{n e}^{*} a_{n e} a_{n p}^{*} a_{n p}$. The induced action of the normalizer on $Z_{n}$ is the group of all permutations. The induced action of the automorphism group consists of the identity transformation and $\zeta_{00}^{n} \stackrel{T_{c}^{n}}{\longrightarrow} \zeta_{00}^{n}, \zeta_{01}^{n} \stackrel{T_{c}^{n}}{\longrightarrow} \zeta_{10}^{n}, \zeta_{10}^{n} \stackrel{T_{c}^{n}}{\longrightarrow} \zeta_{01}^{n}, \zeta_{11}^{n} \stackrel{T_{c}^{n}}{\longrightarrow} \zeta_{11}^{n}$.

In order to give some examples of $\mathfrak{B}_{n}$-faithful invariant states, consider $\mathfrak{A}_{n}$ in its irreducible representation on $C^{4}$. Let $N$ be the linear transformation

$$
N=\left(\begin{array}{cccc}
\lambda_{1} & 0 & 0 & 0 \\
0 & \left(\frac{\lambda_{2}+\lambda_{3}}{2}\right) & \left(\frac{\lambda_{2}-\lambda_{3}}{2}\right) & 0 \\
0 & \left(\frac{\lambda_{2}-\lambda_{3}}{2}\right) & \left(\frac{\lambda_{2}+\lambda_{3}}{2}\right) & 0 \\
0 & 0 & 0 & \lambda_{4}
\end{array}\right)
$$


where $\lambda_{i} \geqq 0$ and $\Sigma \lambda_{i}=1$. Then $\omega_{n}(A)=\operatorname{trace}(N A)$ is an invariant state on $\mathfrak{A}_{n}$. If $\lambda_{1}>0, \lambda_{4}>0$ and either $\lambda_{2}>0$ or $\lambda_{3}>0, \omega_{n}$ will be $\mathfrak{B}_{n}$-faithful. If both $\lambda_{2}>0$ and $\lambda_{3}>0$, then the dimension of the Hilbert space $\mathscr{H}_{n}\left(\zeta^{n}\right)$ on the $n^{\text {th }}$ level of the fibre $\mathscr{S}^{\omega}(\zeta)$ will be four. If either $\lambda_{2}=0$ or $\lambda_{3}=0$, then $\mathscr{H}_{n}\left(\zeta^{n}\right)$ will be three dimensional.

A simple example of a measure quasi-invariant under the action of both the normalizer and the group may be constructed as follows. Let $\mu=\Pi \mu_{n}$ where $\mu_{n}$ is a measure on $Z_{n}$ with nonzero mass at each point and with equal mass at $\zeta_{01}^{n}$ and $\zeta_{10}^{n}$. Then $\mu$ will be quasi-invariant under the action of the normalizer and invariant under the induced action of the group.

ExAmple 3. Let $U_{j}^{n}(g), j=1, \cdots, N(n)$, be a family of finite dimensional representations of some group, $\mathscr{G}$, on spaces $H_{j}^{n}, j=1, \cdots$, $N(n)$. Let $\mathfrak{A}_{n}=\sum_{j=1}^{N(n)} \oplus \mathscr{L}\left(H_{j}^{n}\right)$, where $\mathscr{L}\left(H_{j}^{n}\right)$ denotes the set of all linear transformations on $H_{j}^{n}$. For $A=\bigoplus_{j=1}^{N(n)} A_{j} \in \mathfrak{A}_{n}$ define

$$
\tau_{g}^{n}(A)=\bigoplus_{j=1}^{N(n)} U_{j}^{n}(g) A_{j} U_{j}^{n}\left(g^{-1}\right)
$$

Then $\tau_{g}=\otimes \tau_{g}^{n}$ is a well-defined automorphism group on $\mathfrak{A}=\check{\otimes} \mathfrak{A}_{n}$. Let $P_{j}^{n}$ denote the projection onto $H_{j}^{n}$ and let $\mathfrak{B}_{n}$ be the algebra generated by the $P_{j}^{n}, j=1, \cdots, N(n)$. Notice that $\mathfrak{B}_{n}$ is not maximal abelian but that its normalizer, namely all unitaries of the form $\bigoplus_{j=1} V_{j}^{n}$, generates $\mathfrak{U}_{n}$. Of course, the spectrum, $Z_{n}$, of $\mathfrak{B}_{n}$ consists of exactly $N(n)$ points. The reader can easily investigate for himself the different types of $\check{\otimes} \mathfrak{B}_{n}$-faithful product states which are possible and the structure of the corresponding $\omega$-bundles. Notice that since $\mathfrak{B}$ is left pointwise fixed by both the normalizer and the group there is no induced action on $Z=\times Z_{n}$. Therefore, no quasi-invariance conditions on $\nu$ are necessary and the action of $U_{\nu}^{\omega}(g)$, the unitaries which implement $\mathscr{G}$, all takes place within the fibres.

IV. Crossed products. One cannot expect that the construction in $\S I I$ yields all covariant representations of the system $\left(\mathfrak{A}, \mathfrak{B}, \tau_{\mathscr{g}}\right)$. This is because the fibre spaces are special (infinite tensor product spaces) and the fibre maps for both the group and the algebra are special (they are tensor products of maps operating on different levels of the fibre space). However in certain cases one is guaranteed that all covariant representations are "direct integral representations" over the spectrum of $\mathfrak{B}$ with the relevent operators given by fibre maps. In this section we describe a case when this is so.

Let $\mathscr{G}$ be a group with identity $e$ and $\mathfrak{A}$ a $C^{*}$-algebra with identity I. Suppose that $\mathscr{G}$ is representated as automorphisms of $\mathfrak{A}$, the action being written $g(A)$. One defines the crossed product [4, p. 28] 
of $\mathscr{G}$ and $\mathscr{A}$ as the set of all functions $f: \mathscr{G} \rightarrow \mathfrak{A}$ with finite support subject to the following multiplication

$$
\left(f f^{\prime}\right)(g)=\sum_{h \in \mathscr{G}}(f(h)) h\left(f^{\prime}\left(h^{-1} g\right)\right)
$$

and involution

$$
\left(f^{*}\right)(g)=g\left(f\left(g^{-1}\right)\right)^{*} \text {. }
$$

We write the crossed product as $(\mathscr{G}, \mathfrak{2})$. If $\pi$ is a ${ }^{*}$-algebraic map of $(\mathscr{G}, A)$ into a $C^{*}$-algebra $B$ then one defines a norm on $(\mathscr{G}, \mathfrak{U})$ by $\|f\|=\sup _{\pi}\|\pi(f)\|$. The completion of $(\mathscr{G}, A)$ in this norm is the $C^{*}$-algebra $C^{*}(\mathscr{G}, \mathfrak{2})$. We refer the reader to [4] for various properties of this $C^{*}$-algebra.

In $\S I I$ we considered algebras $\mathfrak{A}=\check{\otimes} \mathfrak{U}_{n}$ where in each $\mathfrak{N}_{n}$ one found a $\mathfrak{B}_{n}$ such that $N\left(\mathfrak{B}_{n}\right)$ spans $\mathfrak{U}_{n}$. We specialize now to the case where each $\mathfrak{A}_{n}$ is a full matrix algebra and $\mathfrak{B}_{n}$ is maximal abelian. Dropping the subscript, suppose then that $\mathfrak{B}$ is generated by the ele-

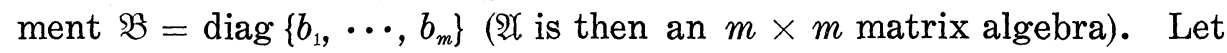
$X=\left\{b_{1}, \cdots, b_{m}\right\}$ and $C(X)$ be the (bounded, continuous) functions on $X$. Consider the abelian group $G$ of cyclic permutations of $m$ elements. The group of course is isomorphic under $\sigma$ with powers of the matrix $C$, where $C$ has ones immediately above the diagonal, $C_{m 1}=1$, and all other entries equal to zero. Each power of the matrix $C$ (up to the $m$-th) is distinct from all previous in that the number one appears only in positions where zeros appeared in previous matrices. The sum of all $m$ powers is the $m \times m$ matrix $\left(a_{j k}\right)$ with $a_{j k}=1$ for all $j, k$. The group $G$ acts as an automorphism of $C(X)$ by $\mathrm{g}(f)(x)=f(g x)$. Returning now to $\mathfrak{N}=\check{\otimes} \mathfrak{N}_{n}$, let $G_{i}, X_{i}$ be the appropriate group and set for the algebra $\mathfrak{A}_{i}$. If $\mathscr{G}$ denotes the weak direct product of the $G_{i}$ and $X$ is the cartesian product of the $X_{i}$ (with the product topology) then one defines the crossed product $(\mathscr{G}, C(X))$ in the obvious manner.

Proposition. Ø̋ $\mathfrak{A}_{n}$ is isomorphic to $C^{*}(\mathscr{G}, C(X))$.

Proof. We follow the method in [4]. If $f_{i} \in C\left(X_{i}\right)$ and $\mathfrak{A}_{i}$ is an $m \times m$ matrix algebra, define a map, $\rho_{i}$, taking $C\left(X_{i}\right)$ onto $\mathfrak{B}_{i} \subseteq \mathfrak{A}_{i}$, by

$$
\rho_{i}\left(f_{i}\right)=\operatorname{diag}\left\{f_{i}\left(b_{1}\right), \cdots, f_{i}\left(b_{m}\right)\right\} \quad \text { where } \quad \mathfrak{B}=\operatorname{diag}\left\{b_{1}, \cdots, b_{m}\right\}
$$

is as in the above discussion. For $g_{i} \in G_{i}$ one has $\rho_{i}\left(g_{i}\left(f_{i}\right)\right)=$ $\sigma_{i}\left(g_{i}\right) \rho_{i}\left(f_{i}\right) \sigma_{i}\left(g_{i}\right)^{-1}$, where $\sigma_{i}$ takes "the permutation $g_{i}$ " onto its corresponding matrix. Forming $\pi_{i}\left(f_{i}\right)=\sum_{g_{i} \in G_{i}} \rho\left(f\left(g_{i}\right)\right) \sigma\left(g_{i}\right)$ we obtain an isomorphism of $C^{*}\left(G_{i}, C\left(X_{i}\right)\right)$ onto $\mathfrak{A}_{i}$. This follows from the "non- 
overlapping" nature of the matrices $\sigma\left(g_{i}\right)$. The proof is then completed by the following string of isomorphisms [4]:

$$
C^{*}(\mathscr{G}, C(X)) \rightarrow C^{*}\left(\mathscr{G}, \check{\otimes} C\left(X_{i}\right)\right) \rightarrow \check{\otimes} C^{*}\left(\mathscr{G}_{i}, C\left(X_{i}\right)\right) \rightarrow \check{\otimes} \mathfrak{A}_{i} .
$$

A different proof of this fact may be found in [15].

This proposition tells us that every cyclic representation of $\check{\otimes} \mathfrak{A}_{i}$ may be realized on a Hilbert space which is a direct integral of Hilbert spaces with respect to some measure on $X$, quasi-invariant under the action of $\mathscr{G}$. For the case of the algebra of anti-commutation relations, this is just the result of Garding and Wightman [3].

V. Infidelity. The construction in $\S I I$ begins with an invariant, $\mathfrak{B}$-faithful product state. The following proposition shows that in the case where $G$ is compact we have not lost the representations which come from invariant, non-faithful product states.

Proposition. Let $\omega^{\prime}=\otimes \omega_{n}^{\prime}$ be an invariant product state. If $\mathscr{G}$ is compact, there is a $\mathfrak{B}$-faithful product state, $\omega=\otimes \omega_{n}$, a quasiinvariant measure $\nu=\Pi \mu_{i}$, and a decomposable operator $T \leftrightarrow T(\zeta)$, such that the representation of $\mathfrak{A}$ generated by $\omega^{\prime}$ through the GNS construction is the cyclic subrepresentation of $\mathfrak{X}$ on the subspace of $L^{2}\left(Z, \nu, \mathfrak{S}^{\omega}(\cdot)\right)$ generated by the section $T(\cdot) \Omega_{0}^{\omega}(\cdot)$ under the action of the operators $\pi_{\nu}^{\omega}(A), A \in \mathfrak{U}$. The operator $T$ may be written as $T(\zeta)=$ $\otimes T_{n}\left(\zeta^{n}\right)$, $\nu$ a.e., where $T_{n}\left(\zeta^{n}\right): \mathscr{H}_{n}\left(\zeta^{n}\right) \rightarrow \mathscr{H}_{n}\left(\zeta^{n}\right)$.

Proof. Each $\omega_{n}^{\prime}$ is invariant under $\tau_{g}^{n}$. If $\omega_{n}^{\prime}$ is $\mathfrak{B}_{n}$-faithful, let $\omega_{n}=\omega_{n}^{\prime}$. If this is not the case we form a new state $\omega_{n}$ which is $\mathfrak{B}_{n}$-faithful and invariant under $\tau_{g}^{n}$. Let $\rho_{n}$ be a state which is faithful on $\mathfrak{B}_{n}$ and $\hat{\rho}_{n}$ an extension to $\mathfrak{A}_{n}$. If $\eta$ is a Haar measure on $\mathscr{G}$, then

$$
\rho_{n}^{\prime}(\cdot)=\int_{\mathscr{f}} \rho_{n}\left(\tau_{g}^{n}(\cdot)\right) d \eta(g)
$$

is an invariant state on $\mathfrak{U}_{n}$. Moreover $\rho_{n}^{\prime}$ is faithful on $\mathfrak{B}_{n}$, since $\tau_{g}^{n}\left(\mathfrak{B}_{n}\right)=\mathfrak{B}_{n}$. The state $\omega_{n}=\alpha \omega_{n}^{\prime}+(1-\alpha) \rho_{n}^{\prime}, 0<\alpha<1$ is then a $\mathfrak{B}$-faithful, invariant state on $\mathfrak{A}_{n}$.

Let $\pi_{\omega_{n}}$ be the representation of $\mathfrak{U}_{n}$, on $H_{n}$, arising via the GNS procedure. If $x_{n}$ is the corresponding cyclic vector then there is a unique $T_{n} \in \mathscr{L}\left(H_{n}\right)$ such that

$$
\begin{gathered}
\omega_{n}^{\prime}(A)=\left(\pi_{\omega_{n}}(A) T_{n} x_{n}, T_{n} x_{n}\right) \quad \text { where } \\
T_{n} \in \pi_{\omega_{n}}\left(\mathfrak{A}_{n}\right)^{\prime} \cap\left\{U_{g}^{n}: g \in \mathscr{G}\right\}^{\prime}, 0 \leqq T_{n} \leqq \frac{I}{\sqrt{\alpha}} \quad[2] .
\end{gathered}
$$


Given $\varepsilon>0$, it is possible to choose $\alpha$ sufficiently close to one so that $\left\|\left(I-T_{n}\right) x_{n}\right\|<\varepsilon$. Let $\delta>0$. Since one may choose $\alpha$ so as to make $\left\|\alpha \omega_{n}^{\prime}-\omega_{n}\right\|<\delta$, one has $\delta>\left|\left(x_{n}, x_{n}\right)-\left(\alpha T_{n}^{2} x_{n}, x_{n}\right)\right|=\left(x_{n}, x_{n}\right)-$ $\left(\alpha T_{n}^{2} x_{n}, x_{n}\right) \geqq 0$, since $0 \leqq \alpha T_{n}^{2} \leqq I$. Then if $\left\{E_{\gamma}^{\alpha}\right\}$ is the spectral family for $\sqrt{\alpha} T_{n}$ one has that

$$
\begin{aligned}
\left(x_{n}, x_{n}\right) \geqq \int_{0}^{I} \gamma d\left(E_{\gamma}^{\alpha} x_{n}, x_{n}\right) \geqq \int_{0}^{I} \gamma^{2} d\left(E_{\gamma}^{\alpha} x_{n}, x_{n}\right) \\
\\
=\left(\alpha T_{n}^{2} x_{n}, x_{n}\right) .
\end{aligned}
$$

Hence $\delta>\left(x_{n}, x_{n}\right)-\left(\sqrt{\alpha} T_{n} x_{n}, x_{n}\right) \geqq 0$. It is clear now that one can find an appropriate $\alpha$, since

$$
\left\|\left(I-T_{n}\right) x_{n}\right\|^{2}=\left(x_{n}, x_{n}\right)-2\left(T_{n} x_{n}, x_{n}\right)+\left(T_{n}^{2} x_{n}, x_{n}\right) .
$$

By varying the $\alpha$ used in the definition of each $\omega_{n}$ we can make $\left\|\left(I-T_{n}\right) x_{n}\right\|$ decrease in norm (as $\left.n \rightarrow \infty\right)$ sufficiently fast so that $T=\otimes T_{n}$ is a well defined operator on the separable subspace of $\otimes H_{n}$ defined by the $c_{0}$-vector $\otimes x_{n}$. We denote this subspace by $H\left(\otimes x_{n}\right)$. Note that $\otimes x_{n}^{\prime}=\otimes T_{n} x_{n} \in H\left(\otimes x_{n}\right)$.

Guichardet has shown [4] that if we decompose each $H_{n}$ into a direct integral with respect to a measure $\mu_{n}$ (as in $\S I I$ ) then $H\left(\otimes x_{n}\right.$ ) is the Hilbert space $L^{2}\left(Z, \nu, \mathfrak{S}^{\omega}(\cdot)\right)$ where $\nu=\Pi \mu_{i}$. This measure is clearly quasi-invariant under the action of $\mathscr{U}$. Moreover, due to the renormalization of $x_{n}\left(\zeta^{n}\right)$ it is invariant under $\tilde{G}$. Thus the theorem of $\S$ II gives us the GNS representation of $\mathfrak{Y}$ due to the tensor product state $\omega=\otimes \omega_{n}$.

Each $T_{n}$ lies in $\pi_{\omega_{n}}\left(\mathfrak{A}_{n}\right)^{\prime}$ and hence

$$
T_{n}=\int_{Z_{n}} T_{n}\left(\zeta^{n}\right) d \mu_{n}\left(\zeta^{n}\right)
$$

We define $T(\zeta)$ on the total subset (8). An element of this set looks like $y(\zeta)=y_{1}\left(\zeta^{1}\right) \otimes \cdots \otimes y_{k}\left(\zeta^{k}\right) \otimes_{n>k} x_{n}\left(\zeta^{n}\right)$. Then

$$
T(\zeta) y(\zeta) \equiv T_{1}\left(\zeta^{1}\right) y_{1}\left(\zeta^{1}\right) \otimes \cdots \otimes T_{k}\left(\zeta^{k}\right) y_{k}\left(\zeta^{k}\right) \otimes_{n>k} x_{n}^{\prime}\left(\zeta^{n}\right)
$$

Clearly $\omega^{\prime}(A)=\left(\pi_{\nu}^{\omega}(A) \otimes x_{n}^{\prime}, \otimes x_{n}^{\prime}\right)$ so that the subspace of $L^{2}(Z$, $\left.\nu, \mathfrak{S}^{\omega}(\cdot)\right)$ for which $T(\cdot) \Omega_{0}^{\omega}(\cdot)$ is cyclic under the action of $\pi_{\nu}^{\omega}(\mathfrak{U})$ is the representation space for the representation of $\mathfrak{U}$ arising from the state $\omega^{\prime}$.

VI. Concluding remarks. $\S 1$. As we mentioned at the start it is possible to extend the analysis of $\S I I$ to the case where $\mathfrak{A}_{i}$ is other than a subalgebra of a matrix algebra. One such possibility is the case where each $\mathfrak{A}_{i}$ is a UHF algebra, viz. there is a sequence $M_{1} \subseteq M_{2} \subseteq \cdots \subseteq M_{n} \subseteq \mathfrak{A}_{i}$, each $M_{i}$ a matrix algebra and $\cup M_{j}$ gen- 
erates $\mathfrak{U}_{i}$. Then if $\mathfrak{B}_{i}$ is an abelian subalgebra of $\mathfrak{U}_{i}$ such that one can extract from $N\left(\mathfrak{B}_{i}\right)$ a subset $\mathscr{F}_{n}$ such that $\operatorname{span}\left\{\mathscr{F}_{n}\right\}=M_{n}$, our analysis goes through. Since $\mathfrak{A}_{i}$ may be written as an infinite tensor product we can use the proposition in $\S$ III to construct such $\mathfrak{B}_{i}$. The reader should consult [12] for related ideas. We thank $R$. Powers for this reference. Certain care must be exercised in choosing the field of Hilbert spaces and the fiber maps to be Borel measurable. This may be done since $\mathrm{sp}\left(\mathfrak{B}_{i}\right)$ is a standard Borel space [7].

The shortcoming of this procedure is that the $\omega$-bundle is no longer defined at every point of $Z$, due to the non-finiteness of $Z_{i}$. In choosing the measure $\nu$ one must be careful not to give positive measure to sets where the bundle is not defined. This will automatically be the case if $\nu$ is absolutely continuous with respect to $\Pi \mu_{i}$.

$\S 2$. Suppose $M$ is a von Neumann algebra acting on a Hilbert space $\mathfrak{S}$. Customary decomposition theory tells us to take an abelian subalgebra in $M^{\prime}$ and use it to decompose $\mathfrak{F}$ rather than, as we have done above, using a subalgebra of $M$. Of course our motivation for the latter was its successful use in [3]. We can, and do, in what follows, make a connection with the usual theory.

We examine one component of the tensor product of $C^{*}$-algebras considered above. Thus let $\mathfrak{A}$ be a $C^{*}$-algebra, (not necessarily finite dimensional) with abelian subalgebra $\mathfrak{B}$, acted upon by a group of automorphisms $\left\{\tau_{g}: g \in \mathscr{G}\right\}$, which preserve $\mathfrak{B}$ and leave a state $\omega$ invariant. We assume that there exists a representation $t \rightarrow \sigma_{t}$ of $\boldsymbol{R}$ as automorphisms of $\mathfrak{X}$ which makes $\omega$ a KMS state [13]. The reader is referred to [6] for a discussion of KMS states on the Clifford algebra. A few remarks about the general theory [13] of such states are in order. If $\omega$ is a faithful normal state on a von Neumann algebra one can always find such a $\sigma_{t}$. For the $C^{*}$-algebra case it follows that the GNS construction yields a representation $\pi_{\omega}$ such that $\omega(A)=$ $\left(\pi_{\omega}(A) \xi_{\omega}, \xi_{\omega}\right)$ where $\xi_{\omega}$ is cyclic and separating for $M=\pi_{\omega}(\mathfrak{Z})^{\prime \prime}$. Moreover there exists an anti-unitary involution $J$ such that $J \xi_{\omega}=\xi_{\omega}$ and $J M J=M^{\prime}$. The automorphism $\sigma_{t}$ lifts to an automorphism $\sigma_{t}^{\omega}$ of $M$.

Let $\mathscr{H}_{\omega}$ be the Hilbert space of the representation $\pi_{\omega}$ and suppose that $\mathscr{H}_{\omega}$ is separable. Above, we "disintegrated" $\mathscr{H}_{\omega}$ by diagonalizing $\sqrt{5}=\pi_{\omega}(\mathfrak{B})^{\prime \prime}$. Could we do just as well with an algebra in $M^{\prime}$ ? The answer is clearly yes. We could use $\widetilde{\mathfrak{S}}=J \varsigma_{5}$ and obtain an equivalent [9] decomposition. The point is that under an additional restriction, the canonical procedure in [9] gives identical decompositions. We thank M. Takesaki for pointing this out.

The condition we need is that $\sigma_{t}^{\omega}(\mathfrak{C})=\mathfrak{C}$. In this case one has $\left[\widetilde{\Im} \xi_{\omega}\right]=\left[J \Subset J \xi_{\omega}\right]=\left[J \Im \xi_{\omega}\right]=\left[\Im \xi_{\omega}\right]$ where the last equality follows from the invariance of $[5$ and is shown for instance in [14]. By using a self-adjoint operator, $A$, which generates $\mathfrak{F}$, we obtain an "ordered 
representation [14] for $\mathscr{H}_{\omega}$ relative to $\mathfrak{E}$, viz, there is a unitary map $W$ from $\mathscr{H}_{\omega}$ onto $\Sigma \oplus L_{2}\left(e_{n}, \mu\right)$ where the $e_{n}$ are a decreasing sequence of Borel sets with $e_{1}$ the real axis. The measure $\mu$ is a finite, regular Borel measure and $W \Subset W^{-1}$ is the algebra of all operators $\left\{f^{n}(\lambda)\right\} \rightarrow$ $\left\{f(\lambda) f^{(n)}(\lambda)\right\}$, where $f(\cdot)$ is an arbitrary bounded Borel function of a real variable.

One then passes canonically to a direct integral representation of $\mathscr{H}_{\omega}$ using the measure $\mu$. An examination of the construction of the ordered representation above reveals that it is (up to complex conjugation) an ordered representation for $\widetilde{\mathfrak{c}}$. This of course use the fact that $\left[\widetilde{\mathfrak{C}} \xi_{\omega}\right]=\left[\mathfrak{\mathbb { F }} \xi_{\omega}\right]$. Thus one obtains canonically the same direct integral decomposition of $\mathscr{H}_{\omega}$ using $\mathbb{E}$ or $\widetilde{\mathfrak{C}}$.

The above brings up the question, of some independent interest, as to when $\sigma_{t}^{\omega}(\mathfrak{C})=\mathbb{C}$. Let us suppose then that $M$ is a factor. Since $\sigma_{t}^{\omega}, t \in \boldsymbol{R}$ and $\tau_{g}, g \in \mathscr{G}$ both leave the state $\omega$ (extended to $M$ ) invariant [13], it follows [6] that $\sigma_{t}^{\omega}$ and $\tau_{g}$ commute as automorphisms of $M$. If $\left(\mathbb{S}\right.$ is the fixed point algebra for $\tau_{g}, g \in \mathscr{G}$ then $\sigma_{t}^{\omega}$ preserves $\sqrt{5}$, since $\tau_{g} \circ \sigma_{t}^{\omega}(C)=\sigma_{t}^{\omega} \circ \tau_{g}(C)=\sigma_{t}^{\omega}(C)$ for all $C \in \mathbb{E}$. With more explicit knowledge of the unitaries, $U_{g}$, implementing $\tau_{g}$ we could give further conditions that $\sigma_{t}^{\omega}(\mathbb{S})=\mathbb{(}$.

Acknowledgment. The authors would like to thank A. S. Wightman and C. Newman for helpful conversations. One of us (RHH) would like to thank E. M. Stein for the opportunity of visiting at Princeton during which time this work was done.

\section{REFERENCES}

1. J. Dixmier, Les algebres d'opérateurs dans l'espace hilbertien, Gauthier Villars, Paris 2é edition, 1969.

2. L_L Les $C^{*}$-algebres et leurs representation, Gauthier Villars, Paris, 1964.

3. L. Garding and A. S. Wightman, Representations of the anticommutation relations, Proceedings of the National Academy of Sciences, 40 (1954), 617-621.

4. A. Guichardet, Produits tensoriels infinis et representation des relations d'anticommutation, Annales de l'école normale supérieure, 83 (1966), 1-52.

5. Une caractérisation des algèbres de von Neumann discrètes, Bull., Soc. Math. France, 89 (1961), 77-101.

6. R. Herman and M. Takesaki, States and automorphism groups of operator algebras, Comm. Math. Phys., 19 (1970), 142-160.

7. G. W. Mackey, The theory of group representations, Chicago lecture notes, 1955.

8. M. Reed, Torus invariance for the Clifford algebra, II, J. Functional Analysis, 8 (1971), 450-468.

9. J. T. Schwartz, $W^{*}$-algebras, Gordon and Breach, 1967.

10. I. E. Segal, Mathematical problems of relativistic physics, Amer. Math. Soc., 1963.

11. D. Shale and W. F. Stinespring, States of the Clifford algebra, Ann. of Math., 80, $365-381$.

12. Sister Rita Jean Tauer, Maximal abelian subalgebras in finite factors of type II, 
Trans. Amer. Math. Soc., 114 281-308.

13. M. Takesaki, Tomita's theory of modular Hilbert algebras and its applications, Lecture Notes in Mathematics $\$ 128$, Springer-Verlag, 1970.

14. - Conditional expectations in Von Neumann algebras, (to appear).

15. - A liminal crossed product of a uniformly hyperfinite $C^{*}$-algebra by a compact abelian automorphism group, J. Functional Analysis, (1) 7 (1971), 140-146.

16. V. S. Varadarajan, Geometry of Quantum Theory, vol. II, Van Nostrand Reinhold Co., 1970.

Received December 17,1970 . The preparation of this paper was partially supported by NSF Grants \#GP-11475 and \#GP-22592.

University of CALIFornia, Los ANGeles

AND

Princeton University 


\title{
PACIFIC JOURNAL OF MATHEMATICS
}

\section{EDITORS}

\author{
H. SAMELSON \\ Stanford University \\ Stanford, California 94305 \\ C. R. HobBY \\ University of Washington \\ Seattle, Washington 98105
}

J. DugundJI

Department of Mathematics

University of Southern California

Los Angeles, California 90007

RICHARD ARENS

University of California

Los Angeles, California 90024

\section{ASSOCIATE EDITORS}
E. F. BECKENBACH
B. H. NeumanN
F. WOLF
K. YOSHIDA

\section{SUPPORTING INSTITUTIONS}

\author{
UNIVERSITY OF BRITISH COLUMBIA \\ CALIFORNIA INSTITUTE OF TECHNOLOGY \\ UNIVERSITY OF CALIFORNIA \\ MONTANA STATE UNIVERSITY \\ UNIVERSITY OF NEVADA \\ NEW MEXICO STATE UNIVERSITY \\ OREGON STATE UNIVERSITY \\ UNIVERSITY OF OREGON \\ OSAKA UNIVERSITY
}

\author{
UNIVERSITY OF SOUTHERN CALIFORNIA \\ STANFORD UNIVERSITY \\ UNIVERSITY OF TOKYO \\ UNIVERSITY OF UTAH \\ WASHINGTON STATE UNIVERSITY \\ UNIVERSITY OF WASHINGTON
AMERICAN MATHEMATICAL SOCIETY
NAVAL WEAPONS CENTER

The Supporting Institutions listed above contribute to the cost of publication of this Journal, but they are not owners or publishers and have no responsibility for its content or policies.

Mathematical papers intended for publication in the Pacific Journal of Mathematics should be in typed form or offset-reproduced, (not dittoed), double spaced with large margins. Underline Greek letters in red, German in green, and script in blue. The first paragraph or two must be capable of being used separately as a synopsis of the entire paper. The editorial "we" must not be used in the synopsis, and items of the bibliography should not be cited there unless absolutely necessary, in which case they must be identified by author and Journal, rather than by item number. Manuscripts, in duplicate if possible, may be sent to any one of the four editors. Please classify according to the scheme of Math. Rev. Index to Vol. 39. All other communications to the editors should be addressed to the managing editor, Richard Arens, University of California, Los Angeles, California, 90024.

50 reprints are provided free for each article; additional copies may be obtained at cost in multiples of 50 .

The Pacific Journal of Mathematics is published monthly. Effective with Volume 16 the price per volume (3 numbers) is $\$ 8.00$; single issues, $\$ 3.00$. Special price for current issues to individual faculty members of supporting institutions and to individual members of the American Mathematical Society: $\$ 4.00$ per volume; single issues $\$ 1.50$. Back numbers are available.

Subscriptions, orders for back numbers, and changes of address should be sent to Pacific Journal of Mathematics, 103 Highland Boulevard, Berkeley, California, 94708.

\section{PUBLISHED BY PACIFIC JOURNAL OF MATHEMATICS, A NON-PROFIT CORPORATION}

Printed at Kokusai Bunken Insatsusha (International Academic Printing Co., Ltd.), 270, 3chome Totsuka-cho, Shinjuku-ku, Tokyo 160, Japan. 


\section{Pacific Journal of Mathematics}

\section{Vol. 40, No. $2 \quad$ October, 1972}

Louis I. Alpert and L. V. Toralballa, An elementary definition of surface area in $E^{n+1}$ for smooth surfaces...........................

Eamon Boyd Barrett, A three point condition for surfaces of constant mean curvature........................................

Jan-Erik Björk, On the spectral radius formula in Banach algebras ....... 279

Peter Botta, Matrix inequalities and kernels of linear transformations . . . . 285

Bennett Eisenberg, Baxter's theorem and Varberg's conjecture ........... 291

Heinrich W. Guggenheimer, Approximation of curves .............. 301

A. Hedayat, An algebraic property of the totally symmetric loops associated with Kirkman-Steiner triple systems ....................... 305

Richard Howard Herman and Michael Charles Reed, Covariant representations of infinite tensor product algebras ................

Domingo Antonio Herrero, Analytic continuation of inner

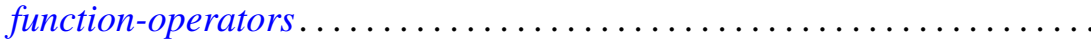

Franklin Lowenthal, Uniform finite generation of the affine group......... 341

Stephen H. McCleary, 0-primitive ordered permutation groups .......... 349

Malcolm Jay Sherman, Disjoint maximal invariant subspaces .......... 373

Mitsuru Nakai, Radon-Nikodým densities and Jacobians .............. 375

Mitsuru Nakai, Royden algebras and quasi-isometries of Riemannian manifolds. . .

Russell Daniel Rupp, Jr., A new type of variational theory sufficiency

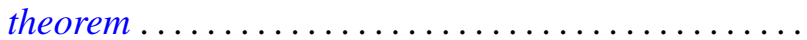

Helga Schirmer, Fixed point and coincidence sets of biconnected multifunctions on trees..........................

Murray Silver, On extremal figures admissible relative to rectangular

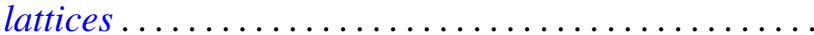

James DeWitt Stein, The open mapping theorem for spaces with unique segments ...

Arne Stray, Approximation and interpolation

Donald Curtis Taylor, A general Phillips theorem for $C^{*}$-algebras and some applications

Florian Vasilescu, On the operator $M(Y)=T Y S^{-1}$ in locally convex algebras...

Philip William Walker, Asymptotics for a class of weighted eigenvalue problems...

Kenneth S. Williams, Exponential sums over $\mathrm{GF}\left(2^{n}\right)$. 